\title{
TESTING THE GRAIN-SIZE DISTRIBUTION DETERMINED BY LASER DIFFRACTOMETRY FOR SICILIAN SOILS
}

\author{
Costanza Di Stefano, Vito Ferro, Stefano Mirabile
}

\section{Introduction}

Particle-size distribution (PSD) is a fundamental physical property which is commonly used for soil classification and for the estimation of some hydraulic properties [Campbell 1992; McCave 1991].

Particles of sand size $(0.05-2.00 \mathrm{~mm})$ are usually determined by sieving while smaller particles are usually determined by classic sedimentation methods as hydrometer or pipette [Gee 1986]. Sieving combined with Hydrometer Method (SHM) has been adopted as an international standard to determine quantitatively the PSD of soils [Allen 1990; Cooper 1984; Liu 1966].

The sedimentation methods are time consuming, especially for the determination of the particles having a size less than $2 \mu \mathrm{m}$, require relatively large samples (10-20 g for the pipette and $50 \mathrm{~g}$ for the hydrometer) and give unreliable results for particles having a size less than $1 \mu \mathrm{m}$ because of the effect of Brownian motion on the rate of sedimentation.

A particle diameter found by Laser Diffractometry Method (LDM) is equivalent to a sphere giving the same diffraction as the particles do. A laser diffraction particle size analyzer sees the particle as a two-dimensional object and gives its grain size as a function of the cross-sectional area of that particle.

Determination of particle-size distribution by LDM has already interested soil scientists [McCave 1986; de Boer 1987; Buurman 1997; Beuselinck 1998; Buurman 2001; Eshel 2004; Pieri 2006] but its application has not replaced the labor-intensive classical methods (pipette (PM) or hydrometer (HM) method) which are considered an international standard for particle-size analysis of soils.

The use of LDM raises the question of how similar

Paper received 24.05.2011; accepted 19.09.2011

Ing. Costanza Di Stefano, Ricercatore, Prof. Vito Ferro, Ordinario di "Idraulica Agraria e Sistemazioni Idraulico-Forestali, Ing. StEFAno Mirabile, collaboratore esterno, Dipartimento dei Sistemi Agro-Ambientali, Università di Palermo, Viale delle Scienze, 90128, Palermo, Italy, vito.ferro@unipa.it

the laser grain-size measurements are to those obtained by a classic technique such as SHM. Buurman [2001] also noted that sand-size particles are measured more or less equally by LDM and Pipette-Sieve Method (PSM) while measurement of the clay-size fraction by LDM usually results in smaller percentages than those obtained by PSM. This means that the lower percentages of the clay fraction measured by LDM must be compensated by higher percentages in silt-size fraction.

Taubner [2009], comparing the pipette method and LDM, concluded that there was no difference detectable in the total sand content between the two methods and negligible differences were detected in the coarse-silt fraction. Clay and the remaining silt fractions showed increasing differences associated with decreasing particle size.

According to Bah [2009] the differences between the two methods are due to the heterogeneity of soil particle density and the deviation of particle shapes from sphericity. Sedimentation methods assume a single particle density, which is a major source of error, whereas LDM is independent of particle density [Goossens 2008]. According to Wen [2002] it may be expected that the stronger the density variations between particles, the larger the difference between the two analyses.

Deviations from sphericity affects both methods. In the case of LDM, an irregular shaped soil particle reflects a cross-sectional area greater than that of a sphere having the same volume. Thus particles become assigned to larger size fraction of the PSD determining an underestimation of the clay fraction. Non-spherical particles in the SHM have longer settling times than their equivalent spheres, which results in overestimation of the clay fraction. Thus, the effect of shape works in opposite direction in LDM and sedimentation method.

Taking into account both that SHM is an accepted and certified method and that LDM provides more information and is more efficient than SHM, a relevant question is to establish whether a correlation exists between fines fractions obtained by both methods.

Recently Di Stefano [2010] tried to solve the ques- 
tion of how similar the laser grain-size measurements are to those obtained by the Sieve-Hydrometer method using 228 samples which were selected to represent a large variety of soil textures based on SHM.

The analysis showed that the sand content determined by SHM is quasi equal to the one obtained by LDM while the so called "overestimation" of clay percentage of SHM with respect to LDM was confirmed. The Authors also demonstrated that for improving the translation of the clay measurements from LDM to SHM two textural groups (sand content greater than $50 \%$ and sand content quasi equal to and less than $50 \%$ ) have to be distinguished.

In this paper, a comparison between the particle size distribution obtained by SHM method and by LDM is carried out. Unlike most of the available literature studies, this paper presents a comparative texture analysis using hydrometer measurements as a reference for LDM.

The analysis is carried out using 747 soil samples, sampled in Sicily, representing a wide range of textures and the Fritsch A22-Economy version laser analyzer.

A set of equations useful to refer laser diffraction measurements to SHM was calibrated using the measurements carried out for 635 soil samples.

Finally, the proposed equations were tested using independent measurements carried out by LDM and SHM for 112 soil samples with a different texture classification.

\section{The Sieving - Hydrometer Method (SHM)}

The hydrometer method defines a particle diameter as equivalent to that of a sphere settling in the same liquid with the same speed as the unknown sized particles, the so-called "Stokes diameter" [Allen 1990; Bernhardt 1994; Konert 1997; Nettleship 1997; Clifton 1999]. The sphere is usually assigned to the density of quartz. The hydrometer analysis uses a hydrometer having a graduated stem and weight bulb, to measure the specific density of the suspension. The specific density depends on the weight of soil particles in the suspension at the time of measurement [Wen 2002].

The hydrometer method is based on Stokes' law that establishes the velocity at which particles settle in suspension assuming that: (1) soil particles are rigid, spherical and smooth; (2) soil particles have similar densities; (3) particle-to-particle interference and boundary effects from the walls of the sedimentation column are negligible; (4) particle sizes are small enough to ensure that the induced fluid flow is well within the laminar flow regime.

Deviations from Stokes' equation are expected when particles are irregular in shape, as most silt particles, or platy or tubular as are most clay particles. The particle-shape effect is due to the circumstance that the most stable position of a settling, non-spherical particle is the one in which the maximum crosssectional area is perpendicular to the direction of motion. As a consequence, this position increases the expected particle drag resistance and reduces the settling velocity. In other words the particle-shape effect results in a so-called "overestimation" of the fine size fraction which depends on at which size platy particles appear.

Recently, Lu [2000] carried out a theoretical analysis for determining the settling velocity of diskshaped and rod-shaped particles. The analysis showed that for disk-shaped and rod-shapes particles, having a size ranging from $0.1 \mu \mathrm{m}$ to $100 \mu \mathrm{m}$, Stokes' law underestimates the maximum particle dimension by up to two orders of magnitude. Experimental results of $\mathrm{Lu}$ [2000], using various techniques, also confirmed the underestimate of particle size inherent in hydrometer analysis.

Particle density of soil minerals affects the sedimentation method only, by means of the settling velocity from Stokes' equation. The mineral composition of soil samples, affecting particle density, is variable over the whole range of particle sizes [Taubner 2009]. Clifton [1999] suggested that density of sediment particles can vary between 1.66 and $2.99 \mathrm{Mg} \mathrm{m}^{-3}$. The uncertainty of the actual particle density may bias the particle size distribution [Wen 2002], [Ferro 2009]. Since no detailed information is available, the density of quartz particle which also represents an average of the common clay minerals in many soils is used as substitute.

The classic technique SHM represents a "standard" for soil particle size analysis and many available relationships, such as pedotransfer functions, were established using hydrometer/pipette texture measurements.

\section{The Laser Diffraction Method (LDM)}

The principle of the Laser Diffraction Method (LDM) is that particles of a given size diffract light through a given angle. The angle of diffraction is inversely proportional to particle size, and the intensity of the diffracted beam at any angle is a measure of the number of particles with a specific cross-sectional area in the beam's path.

A beam of monochromatic light passes through a suspension contained in a sample cell, and the diffracted light is focused onto detectors. For calculating particle sizes from light intensity sensed by detectors, two diffraction theories are commonly used: the Fraunhofer diffraction model and the Mie theory [Di Stefano 2010]. Both theories assume that the particles have a spherical shape; in other words, the particle dimension is the optical spherical diameter, i.e. the diameter of the sphere having a cross-section area equivalent to the measured one by laser diffraction.

The Fraunhofer theory [de Boer 1987; Loizeau 
1994] is based on the approximation that the laser beam is parallel and the detector is at a distance that is very large compared with the size of the diffracting particle. Matrices based on Fraunhofer theory are calculated from diffraction by the particles and differences in absorption and refraction indices have no effect on the calculated grain-size distribution.

The Mie theory is a solution of the Maxwell equations describing propagation of the electromagnetic wave of light in space [Eshel 2004]. The Mie theory takes into account phenomena of transmission through the particle and therefore requires knowledge of the refractive index $R I$ of the tested soil. The refractive index of a material is a function both of particle size and material's composition. Taking into account that soils are generally multisized and polymineralic in nature, this circumstance can make difficult to choose a representative $R I$ for a given soil. The refractive index is a complex number [Eshel 2004] comprised of a real part $n_{r}$, representing the change in the velocity of light through the tested material compared with the velocity of light in vacuum, and an imaginary term $n_{i}$ which represents the transparency and absorptivity of the tested material.

The particle mineralogy affects the refractive index which has a strong influence on the calculated size distribution by LDM [Sperazza 2004]. Eshel [2004] proposed a value of 1.53 as approximation to most soil minerals. Especially the detected clay content may distinctly differ by varying $R I$ values of 1.5 to 1.6.

The LDM analyzes small samples in a short time ( 5 to $10 \mathrm{~min}$ per sample), so it is suitable for a rapid and accurate analysis from a large number of samples (e.g. soil samples sampled in a basin, suspension samples caught during soil erosion events).

LDM covers a wide range of grain sizes and may also be used to analyze non-dispersed samples [Muggler 1997]. Although the fully dispersed size distribution (ultimate grain-size distribution) is important with respect to certain soil chemical and physical properties, other relevant processes, such as soil erosion and sediment transport by overland flow, are dependent on the size distribution of soil aggregates (effective grain-size distribution) [Foster 1985; Di Stefano 2002].

\section{Materials and methods}

Soil samples were taken at various location in Siciliy. The 747 samples were selected to represent a large variety of soil textures based on SHM (Figure 1). Some samples (635) were used to calibrate LDM and 112 sample were used to test the set of equations useful to refer laser diffraction measurements to SHM.

For both SHM and LDM, soil samples were dried at $105{ }^{\circ} \mathrm{C}$ and were gently crushed and dry sieved at 2 - mm mesh-size. For each analyzed soil sample, $50 \mathrm{~g}$ were used for SHM analysis and $10 \mathrm{~g}$ for LDM. Each sample was treated with $\mathrm{H}_{2} \mathrm{O}_{2}$ (concentration equal to
$30 \%$ ) to assure complete removal of organic material and was dispersed to remove aggregates by adding a sodium hexametaphosphate solution over night [Gee 2002]. A volume of $100 \mathrm{ml}$ of sodium hexametaphosphate solution, having a concentration equal to $50 \mathrm{~g} \mathrm{l}^{-1}$, was used. The treated sample was mixed overnight using an end - over - end shaking.

For SHM analysis the pretreated sample $(50 \mathrm{~g})$ was wet sieved through a $0.075 \mathrm{~mm}$ sieve. The fine fraction $(<75 \mu \mathrm{m})$ collected after wet sieving was transferred to standard cylinders for hydrometer analysis. The cylinders were inserted into a tank full with water having a constant temperature. Corrections for the temperature effects on density and viscosity of suspension were carried out. A standard hydrometer, ASTM no. $152 \mathrm{H}$, with Bouyoucos scale $\left(\mathrm{g} \mathrm{l}^{-1}\right)$ was used. The suspension was mixed using an end-overend shaking for $1 \mathrm{~min}$ [Gee 2002]. The hydrometer analysis was carried out by multiple readings at 2,5 , $15,30,60,180,1440$ and $2880 \mathrm{~min}$ [Gee 2002]. The coarse fraction retained by the $0.075 \mathrm{~mm}$ sieve was oven-dried at $105^{\circ}$, weighed and dry sieved at 0.075 , $0.106,0.250,0.425,0.85$ and $2 \mathrm{~mm}$. The adopted sieve sizes belong to the series R $40 / 3$ of the standard ISO 3310-1.

For LDM analysis, in the range $0.1-600 \mu \mathrm{m}$, the pretreated sample $(10 \mathrm{~g})$ was firstly wet sieved through a $710 \mu \mathrm{m}$ sieve. A pretreated sub-sample, having a volume of $1.5 \mathrm{ml}$, was introduced into the dispersion unit device of the laser particle analyzer, that contained $400 \mathrm{ml}$ of deionized water, for the measurement.

In this investigation the FRITSCH Laser Particle Sizer Analysette 22 - Economy version was used. This instrument measures 31 grain-size classes in the working range of $0.1-600 \mu \mathrm{m}$. For the LDM analysis the sub-sample is introduced into the dispersion unit device where, to maintain the random orientation

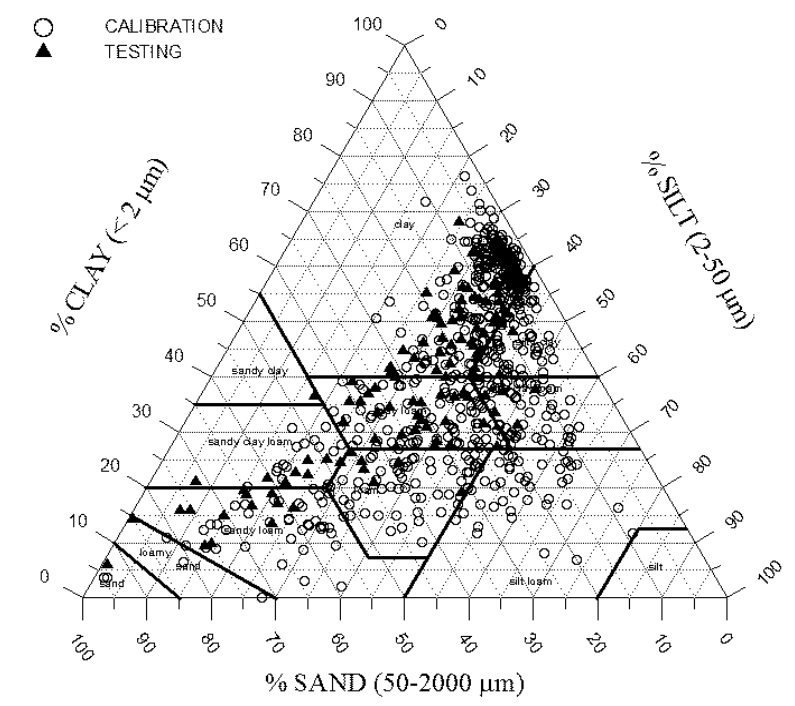

Fig. 1 - Distribution by USDA texture using the percentage of clay, sand and silt determined by SHM. 
of particles in suspension, automatic ultrasonic function is applied during the run.

The ultrasonic action is an efficient dispersion method but can be critical for the particle size distribution because although the clay coatings are quickly removed the quartz grain can be o broken up. According to Chappel [1998] a 3-min duration of ultrasonic action was used in the runs.

To prevent the formation of gas bubbles during the movement of suspension into the dispersion unit device, the stirrer velocity was set to 60-70 revolutions/s.

The suspension was then pumped through a sample cell placed in the convergent laser beam where the forward scattered light fell onto the 31 photosensitive sensor rings. Each run was set for $60 \mathrm{~s}$.

Prior to each run, the detectors were aligned, the background measured and the sample dilution controlled (to test that the used sub-sample volume allows a correct analysis). All operations were controlled by a personal computer.

\section{Results}

At first, for testing the effect of the particle shape, SHM and LDM were compared using two artificial samples (M1 and M2). These two samples were real-
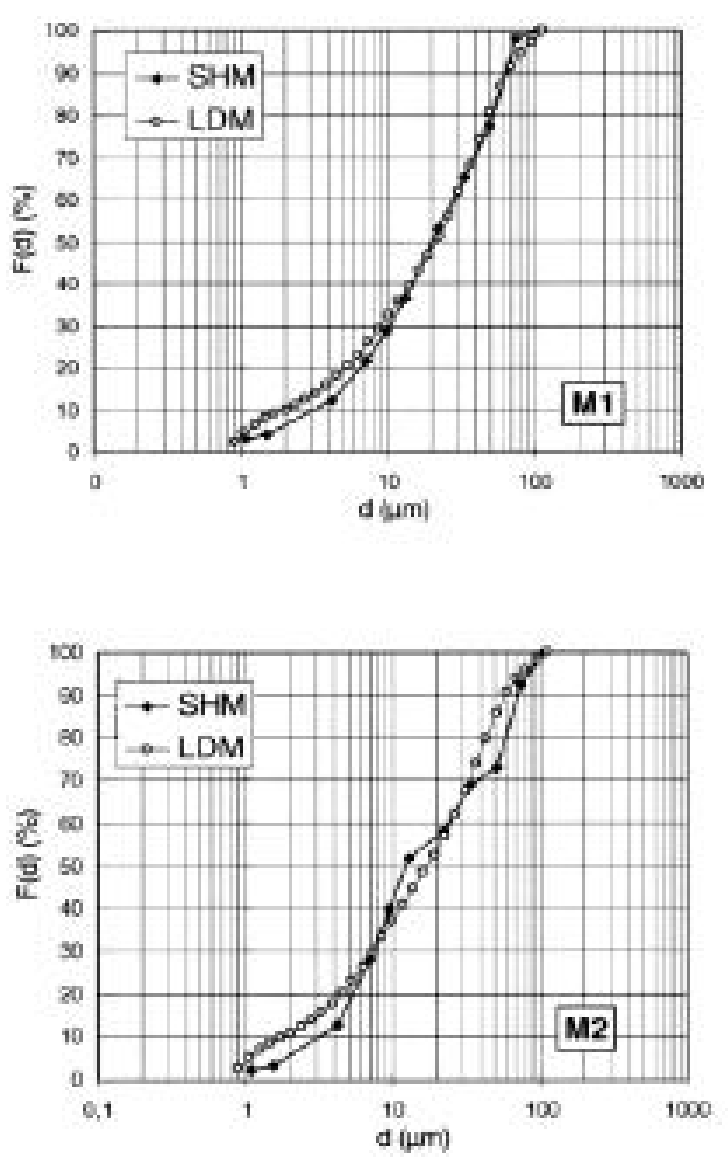

Fig. 2 - Comparison, for the M1 and M2 samples, of the cumulative particle size distributions obtained by SHM and LDM methods. ized using four samples of glass sphere type Speriglass ${ }^{\circledR}$ (soda-lime-silica glass) produced by Potter Industries. The four industrial samples, named 2000 (sphere having a diameter equal to $24,45,63$ and 100 $\mu \mathrm{m}), 2530$ (45, 63, 90 and $106 \mu \mathrm{m}), 3000$ (24, 45, 63 and $100 \mu \mathrm{m})$ and $5000(12,24$ and $45 \mu \mathrm{m})$, are characterized by both a constant bulk density (equal to $2.5 \mathrm{~g}$ $\mathrm{cm}^{-3}$ ) and a refraction index equal to $1.50-1.53$. Industrial samples having the same particle diameters (2000 and 3000) are characterized by a different proportion of each diameter class.

The mix M1 was realized using equal part of the four glass sphere samples $(2000,2530,3000$ and 5000) while the mix M2 was realized using equal part of the two samples 2530 and 5000.

Figure 2 shows the comparison, for the M1 and M2 samples, of the cumulative particle size distributions obtained by SHM and LDM. This figure demonstrates that the two grain-size distributions are overlaid and therefore no difference is appreciable between the two measurement techniques when the bulk density is constant and the shape of the particle is spherical.

Results obtained in a previous paper [Di Stefano 2010] showed that using the two diffraction models (Fraunhofer and Mie) no appreciable differences can be detected into the grain-size distributions for 228 investigated Sicilian soil samples. For the same soil samples, the analysis showed that the variability of the imaginary term of the refraction index $(0.1-0.2)$ does not produce appreciable effects on the cumulative grain size distribution.

According to these results, the cumulative grainsize distributions of the investigated samples were determined using the Fraunhofer diffraction model.

Figure 3, which compares the sand content determined with SHM, named $S A_{S H M}$, with the Laser Diffraction measured sand content, $S A_{L D M}$, shows that the two percentages can be assumed equal:

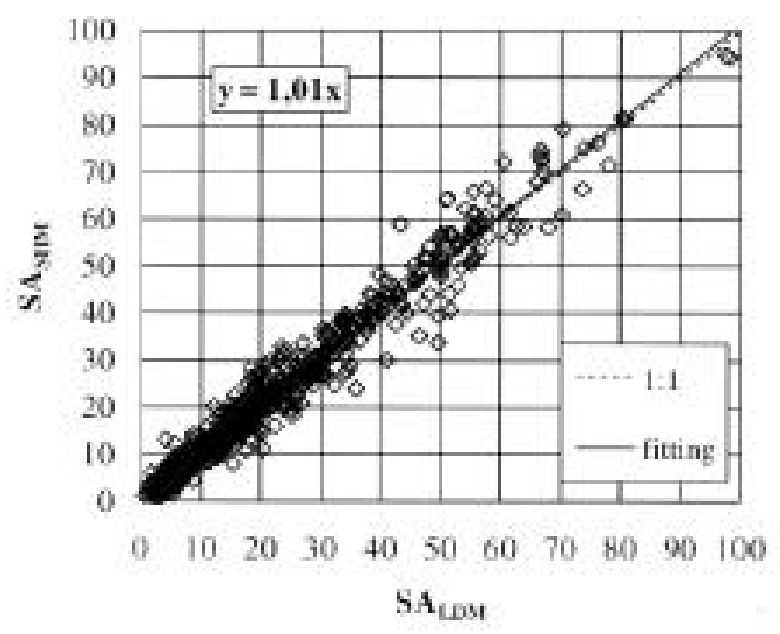

Fig. 3 - Relationship between sand fraction SA obtained by LDM and the one measured by SHM. 


$$
S A_{S H M} \cong S A_{L D M}
$$

The relationship plotted in Figure 3 is characterized by a root mean square error RMSE equal to 3.3 (expressed as percentage).

The direct measurement of particle size by sieving and the calculated equivalent spherical hydraulic diameter by hydrometer method, and optical spherical diameter by LDM are incompatible in essence [Wen 2002]. Therefore, the good agreement between SHM and LDM for sand fraction may indicate that a particle's true intermediate size is close to its optical spherical diameter.

For the clay fraction the following equation can be established:

$$
C L_{S H M}=a C L_{L D M}
$$

in which $C L_{S H M}$ and $C L_{L D M}$ are, respectively, the clay percentage determined by Sieve Hydrometer and Laser Diffraction Methods (Figure 4) and $a$ is a calibration coefficient which can be assumed equal to 2.07 for the complete data set. The relationship plotted in Figure 4 is characterized by a root mean square error RMSE equal to 9.5 (expressed as percentage). The so called "overestimation" of the clay percentage measured by SHM as compared to LDM, which si recognized by most of the literature studies, is confirmed.

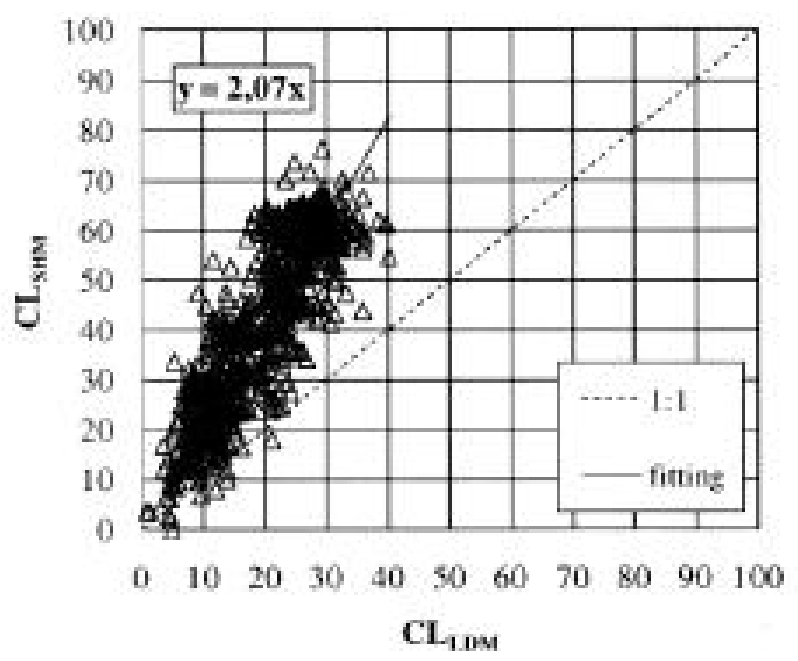

Fig. 4 - Relationship between clay fraction CL obtained by LDM and the one measured by SHM.

This result disagrees only with the analysis by Goossens [2008] which, using the FRITSCH Laser Particle Sizer Analysette 22 and loamy sediments (sediment particles with a diameter less than $90 \mu \mathrm{m}$ ), established that laser diffraction systematically overestimated the clay content compared with the pipette method. Goossens' result is strongly affected by the type of analyzed sediments in which no coarser particles are present.
The analysis showed that there is a good statistically significant correlation between particle size distribution measured by aerometric and laser diffraction methods [Ryzak 2010] and the correlation for coarser fraction (sand) is better than for the finer fraction (clay).

Using eqs. (1) and (2) allows to obtain the following estimate, $S I_{E}$, of silt percentage:

$$
S I_{E}=100-a C L_{L D M}-S A_{L D M}
$$

Taking into account that SHM has been adopted as an international standard to determine quantitatively the PSD of soils [Cooper et al. 1984], the use of eqs. (1) and (2) allow to refer the sand and clay percentage measured by LDM to SHM standard.

For deriving equations useful to translate measurements from LDM to SHM, the comparison between LDM and SHM measurements was also carried out grouping soils with respect to their USDA classification. In particular, for comparing the $C L$ percentage measured by the two different methods the soil samples were examined taking into account their sand content $S A$.

In particular, the calibration coefficient $a$ can be estimated by the following relationships (Figure 5):

$$
\begin{array}{ll}
a=2.07 & \text { for } \mathrm{SA} \leq 35 \% \\
a=1.6669+0.0258 \mathrm{SA}-0.0004 S A^{2} & \text { for } 35 \%<\mathrm{SA} \leq 70 \%
\end{array}
$$

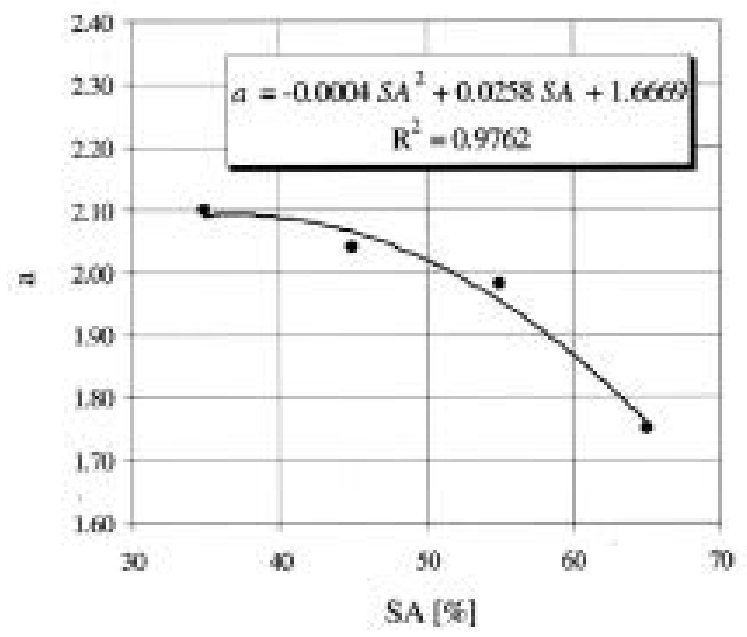

Fig. 5 - Relationship between the coefficient $a$ and the sand content in the range $35 \%<\mathrm{SA} \leq 70 \%$.

The calibration equation (2), with $a=2.07$ for $S A$ less than or equal to $35 \%$ and estimated by eq.(5) for sand content $35 \%<S A \leq 70 \%$, was finally tested using independent measurements carried out by LDM and SHM for 112 soil samples with a different texture classification.

Figure 6 shows, for the 112 soil samples, the comparison between the clay fraction $C L_{S H M}$ measured by SHM and the one estimated by eq. (2). This compari- 


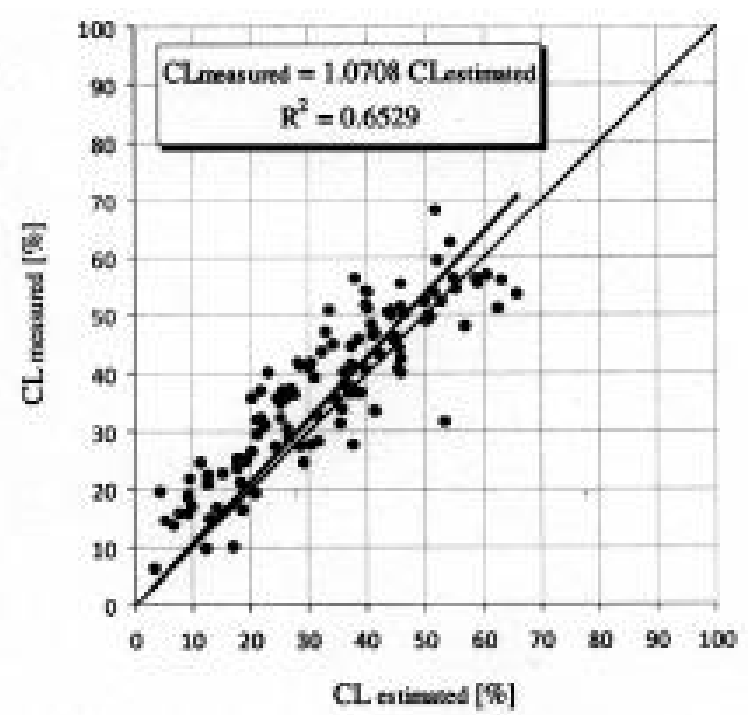

Fig. 6 - Comparison between clay fractions obtained by LDM and the ones measured by SHM for the testing group.

son shows that the measured $C L_{S H M}$ values are quasi equal to the estimated ones (the linear correlation is characterized by a slope equal to 1.07) even if the scattering of the pairs is appreciable.

The distinct deviation between SHM and LDM for silt and clay fractions in the samples may be largely credited to their polymineralic compositions, including quartz, sesquioxides and clay minerals. Particularly, sesquioxides may substantially increase density of clay-sized grains by coating and binding them.

The calibration equation (2) presented in this paper is not universal. As stated earlier, correlations of the laser diffraction method with the sieving-hydrometer method may vary for a variety of reasons related to used laser diffraction analyzer, particle shape, mineralogy, refractive index, etc. Poor correlations of laser diffraction results with sieving-hydrometer may result when attempting to extend calibration equation (2) to other areas.

\section{Conclusions}

Taking into account that LDM provides more information and is more efficient than SHM while the latter is an accepted and certified method, this paper tried to solve the question of how similar the laser grain-size measurements are to those obtained by a classic technique such as Sieve-Hydrometer method.

This study was developed using 747 soils sampled in Sicily, having a variety of texture and the Fritsch A22-Economy version laser analyser.

The results obtained in this paper have to be considered apparatus specific because the measurement accuracy is dependent on the number of detection cells.

This study showed that the sand content determined by SHM is quasi equal to the one obtained by
LDM while the so called "overestimation" of clay percentage of SHM with respect to LDM is confirmed.

The good agreement between the SHM and LDM for sand fraction may indicate that a particle's true intermediate size is close to its optical spherical diameter.

The distinct deviation between SHM and LDM for silt and clay fractions in the samples may be largely credited to their polymineralic compositions, including quartz, sesquioxides and clay minerals.

Variations in particle density, shape and mineralogy of sand, silt and clay size ranges are the major factors leading to the differences between grain-size distribution obtained by SHM and LDM.

Finally, an equation useful to refer the Laser Diffraction measurements to the Sieve-Hydrometer method, which is used as an international standard, was also proposed.

The analysis demonstrated that for improving the translation of the clay measurements from LDM to SHM, the relationship between the calibration coefficient and the soil sand content has to be used. This relationship was finally tested using independent measurements carried out by LDM and SHM for 112 soil samples.

Even if for translating clay measurements from LDM to SHM a more reliable estimate of clay percentage can be obtained using a calibration coefficient specific for each textural group; the calibration equations (4 and 5 ) by particle size (SA $<35 \%$ and $35 \%<$ $S A \leq 70 \%)$ presented in this paper are not universal. Therefore, for the determination of soil texture of unknown soil samples by LDA-derived and by regression-transformed size fractions, a validation with SHM is essential.

Further measurements contemporaneously carried out by Laser Diffraction and Sieve-Hydrometer methods will allow both to confirm the results obtained in this investigation and to improve the proposed scale equations.

\section{Acknowledgements}

The research was set up by Prof. V. Ferro and Dr. C. Di Stefano, the measurements were carried out by Dr. S. Mirabile. All authors analyzed the results and contributed to the writing of the paper.

The research was supported by Progetto MOFEROS.

\section{References}

Allen T.A., Particle Size Measurement. 4th ed., Chapman and Hall, 1990, London.

Bah A. R., Kravchuk O., Kirchhof G., Fitting performance of particle-size distribution models on data derived by conventional and laser diffraction techniques. Soil Science Society America Journal, 2009, 73, 1101-1107. 
Bernhardt C., Particle Size Analysis : Classification and Sedimentation Methods. Chapman and Hall, 1994, London, UK.

Beuselinck L., Govers G., Poesen J., Degraer G., Froyen, L., Grain-size analysis laser diffractometry: Comparison with the sieve-pipette method. Catena, 1998, 32, 193208.

Buurman P., Pape Th., Muggler C.C., Laser grain-size determination in soil genetic studies. 1. Practical problems. Soil Science, 1997, 162, 211-218.

Buurman P., Pape Th., Reijneveld J.A., de Jong F., van Gelder E., Laser-diffraction and pipette-method grain sizing of Dutch sediments: correlations for fine fractions of marine, fluvial and loess samples. Netherlands Journal of Geosciences, 2001, 80, 49-57.

Campbell G.S., Shiozawa S., Prediction of hydraulic properties of soils using particle-size distribution and bulk density data. In: van Genuchten M.T., Leij F.J., Lund L.J. (Eds.). Indirect methods for estimating the hydraulic properties of unsatured soils, University of California, 1992, Riverside, 317-328.

Chappel A., Dispersing sandy soil for the measurement of particle size distributions using optical laser diffraction. Catena, 1998, 31, 271-218.

Clifton J., McDonald P., Plater A., Oldfield F., An investigation into the efficiency of particle size separation using Stokes' Law. Earth Surface Processes and Landforms, 1999, 24, 725-730.

Cooper L.R., Haverland R.L., Hendricks D.M., Knisel W.G., Microtac particle-size analyzer: An alternative particle-size determination method for sediment and soils. Soil Science, 1984, 138, 138-146.

CRC Press., Handbook of chemistry and physics. $83^{\text {rd }}$ Ed. CRC Press, 2002, Boca Raton FL.

de Boer G.B., de Weerd C., Thoenes D., Goossens H.W., Laser diffraction spectrometry: Fraunhofer versus Mie scattering. Particle Characteristics, 1987, 4, 14-19.

Di Stefano C., Ferro V., Linking clay enrichment and sediment delivery processes. Biosystems Engineering, 2002, 81, 465-47.

Di Stefano C., Ferro V., Mirabile S., Comparison between grain-size analyses using laser diffraction and sedimentation methods. Biosystems Engineering, 2010, 106, 205-215.

Eshel G., Levy G.J., Mingelgrin U., Singer M.J., Critical evaluation of the use of laser diffraction for particle-size distribution analysis. Soil Science Society America Journal, 2004, 68, 736-743.

Ferro V., Mirabile S., Comparing particle size distribution analysis by sedimentation and laser diffraction method. Journal of Agricultural Engineering, 2, 35-43.

Foster G.R., Young R.A., Neibling W.H., Sediment composition for nonpoint source pollution analyses. Transactions of the ASAE, 1985, 28, 133-146.

Gee G.W., Bauder J.W., Particle-size analysis. In: J. H. Klute (Ed.) Methods of soil analysis. Part 1. 2nd Ed. Agron. Monogr. 9. ASA and SSSA, 1986, Madison, WI, 383-411.

Gee G.W., Or D., Particle-size analysis. In: J.H. Dane and G.C. Topp (Eds.) Methods of soil analysis. Part 4. Physical Methods. Soil Science Society of America Book Series 5, 2002, Madison, WI, 255-293.

Goossens D., Techniques to measure grain-size distributions of loamy sediments: a comparative study of ten instruments for wet analysis. Sedimentology, 2008, 55, 65-96.

Konert M., Vandenberghe J., Comparison of Laser Grain Size Analysis with Pipette and Sieve Analysis: A Solution for the Underestimation of the Clay Fraction. Sedimentology, 1997, 44, 523-525.

Liu T.K., Odell R.T., Etter W.C., Thornburn T.H., Comparison of clay contents determined by hydrometer and pipette methods using reduced major axis analysis. Soil Science Society America Proceedings, 1966, 30, 665669.

Loizeau J.L., Arbouille D., Santiago S., Vernet J.P., Evaluation of a wide range laser diffraction grain size analyzer for use with sediments. Sedimentology, 1994, 41, 353361.

Lu. N., Ristow G. H., Likos W. I., The Accuracy of Hydrometer Analysis for Fine-Grained Clay Particles. Geotechnical Testing Journal, 2000, 23, 487-495.

McCave I. N., Bryant R.J., Cool H.F., Coughanowr C.A., Evaluation of a laser-diffraction-size analyser for use with natural sediments. J. Sedimentol. Petrol., 1986, 56, 561-564.

McCave I. N., Syvitski J. P. M., Principles and Methods of Geological Particle Size Analysis. In: Principles, Methods and Application of Particle Size Analysis, J. M. P. Syvitski, Ed., Cambridge University Press, 1991, Cambridge, UK, 3-21.

Muggler C.C., Pape Th., Buurman P., Laser grain-size determination in soil genetic studies: 2 . Clay content, clay formation, and aggregation in some Brasilian Oxisols. Soil Science, 1997, 162, 219-228.

Nettleship I., Cisko L., Vallejo L. B., Aggregation of clay in the hydrometer test. Canadian Geotechnical Journal, 1997, 34, 621-626.

Pieri L., Bittelli M., Rossi Pisa P., Laser diffraction, transmission electron microscopy and image analysis to evaluate a bimodal Gaussian model for particle size distribution in soils. Geoderma, 2006, 135, 118-132.

Ryźak M. Bieganowski A., Determination of particle size distribution of soil using laser diffraction comparison with aerometric method. Int. Agrophysicis, 2010, 24 177-181.

Sperazza M., Moore J.M., Hendrix M.S., High-resolution particle size analysis of naturally occurring very finegrained sediment through laser diffractometry. J. Sediment. Res., 74, 736-743.

Taubner H., Roth B., Tippkötter R., Determination of soil texture: comparison of the sedimentation method and the laser-diffraction analysis. J. Plant Nutr. Soil Sci., 2009 172, 161-171.

Wen B., Aydin A., Duzgoren-Aydin N.S., A comparative study of particle size analyses by sieve-hydrometer and laser diffraction methods. Geotechnical Testing Journal, $2002,25,434-442$.

\section{SUMMARY}

In this paper the soil grain-size distribution determined by Laser Diffraction method (LDM) is tested using the Sieve-Hydrometer method (SHM) applied for 747 soil samples representing a different texture classification, sampled in Sicily. 
The analysis showed that the sand content measured by SHM can be assumed equal to the one determined by LDM.

An underestimation of the clay fraction measured by LDM was obtained with respect to the SHM and a set of equations useful to refer laser diffraction measurements to SHM was calibrated using the measurements carried out for 635 soil samples.
Finally, the proposed equations were tested using independent measurements carried out by LDM and SHM for 112 soil samples with a different texture classification.

Keywords: Particle-size distribution, Sieve-Hydrometer method, Laser Diffraction method. 
The new functionalities of www.aiia.it

The Italian Association of Agricultural Engineering has a presence on the World Wide Web since 2002, thanks to prof. Ettore Gasparetto. In the subsequent years the groups of Bologna and Milan have been responsible for maintaining the hardware and software structure, updating constantly the content according to the needing of AIIA presidents.

Recently this activity has received a new impulse from prof. Alessandro Santini, through a complete redesign of the setup, looking for a greater interface usability, easier updating of content and easier management of the ever growing database.

The first evidence of this transformation has been the moving from domain www.aiia.info toward the new www.aiia.it that has recently become available. It should be noted that the old domain aiia.info will remain online for few months, until the contract will expires. An automatic redirect is actually working for those who still connect to the old address.

The figure 1 shows the appearance of the new homepage, where the central space is dedicated to the most recent information. In the following text is reported a brief showcase of the most relevant features of the new AIIA portal.

(1) it is possible to translate in the main European languages the content of every page, using the small rectangular flag icons in the upper right corner. This is an automatic translation not very reliable, however capable to continuously adapt himself to the modifications in the pages content and will be probably sufficiently useful for international visitors.

Along the same line is available the banner "AIIA - Italian Society of Agricultural Engineering". This provide an active link that allows the user to return to the homepage from any internal page.

(2) On the left side there is a quick links column. The first link "Home" has the same goal of the banner "AIIA" in the top of the webpage.

The "Archivio News" (News Archive) opens an informative section, where the most important communications to AIIA members are stored. E.g. are reported news about periodic AIIA meetings or scientific conferences. Particularly the news are divided as information directly related to AIIA activity (AIIAnews) and others (AltreNews) such as CIOSTA conferences.

The "Journal of Agricultural Engineering" link points to specific web pages of the Journal of the Association: www.jae.unibo.it.

The fourth link "Storico Convegni AIIA" includes a list of the AIIA conferences held since 1966. Particularly, for the last one held in Ischia in September 2009, are stored even all the documents (in PDF format) divided by sessions.

All the stored documents are being indexed by the major search engines. As a consequence these papers, even in Italian, will gain a greater visibility.

Looking ahead, the webspace aiia.it could provide a base for the conservation of proceedings of next AIIA meetings.

The box below, "Calendario Convegni" (Meetings Schedule), is related to the AIIAnews section providing a useful visual representation of it. AIIA related events, past and planned, are shown on a monthly calendar.

It is sufficient to pass on the colored checkboxes with the mouse pointer to open a floating window that contains the specific information. One click leads on active link bring directly to the related page. As an example is possible to experience operating on April 2011.

The last box in the lower side provides specific areas for each of the seven sections of AIIA.

(3) this menu line it is probably self-explanatory. The first item "Storia" contains a chronology of AIIA activities since the foundation. The sections "Subscriptions" refer to the registration of new members (individual or collective), while "Struttura Organizzativa" describe the current composition of the board, the statute and rules of the Association. Moreover it is possible to search inside the directory of AIIA members. The item "Convegni" is obviously linked to the news section, being an archive of conferences of interest for AIIA. The interface of this section, very intuitive, allows some practical functions of sorting and searching. 
The item "Links Utili" (Useful Links) is designed to quickly connect aiia.it to other web structures of similar national and international associations: CIGR, EurAgEng and ISSA. Finally, the "Documenti" (Documents) section contains all the downloadable materials available on the site. This material is distributed across different pages and is referred from other part of the web structure, but could it be useful to find it quickly as aggregate.

(4) Among other features, that we invite to explore, we would highlight the internal search engine (see the small window "search" on the right side) and the "site map" link which shows (bottom right) a summary of the structure of the website dynamically generated, being so automatically updated.

All features are fairly intuitive to use, as a consequence we do not want insist here on deep details, considering sufficient just some direct experience. It with a group of tools whose operating modes are now part of the professional practice for all of us.

The developing and feeding of aiia.it is going on with the aim to offer new services, such as a periodical newsletter.

As a conclusion aiia.it is currently a versatile platform, ready to host and organize material that AIIA members will consider of general interest for association.

Angelo Fabbri

University of Bologna

afabbri@agrsci.unibo.it
Fabrizio Sarghini

University of Napoli

fabrizio.sarghini@unina.it

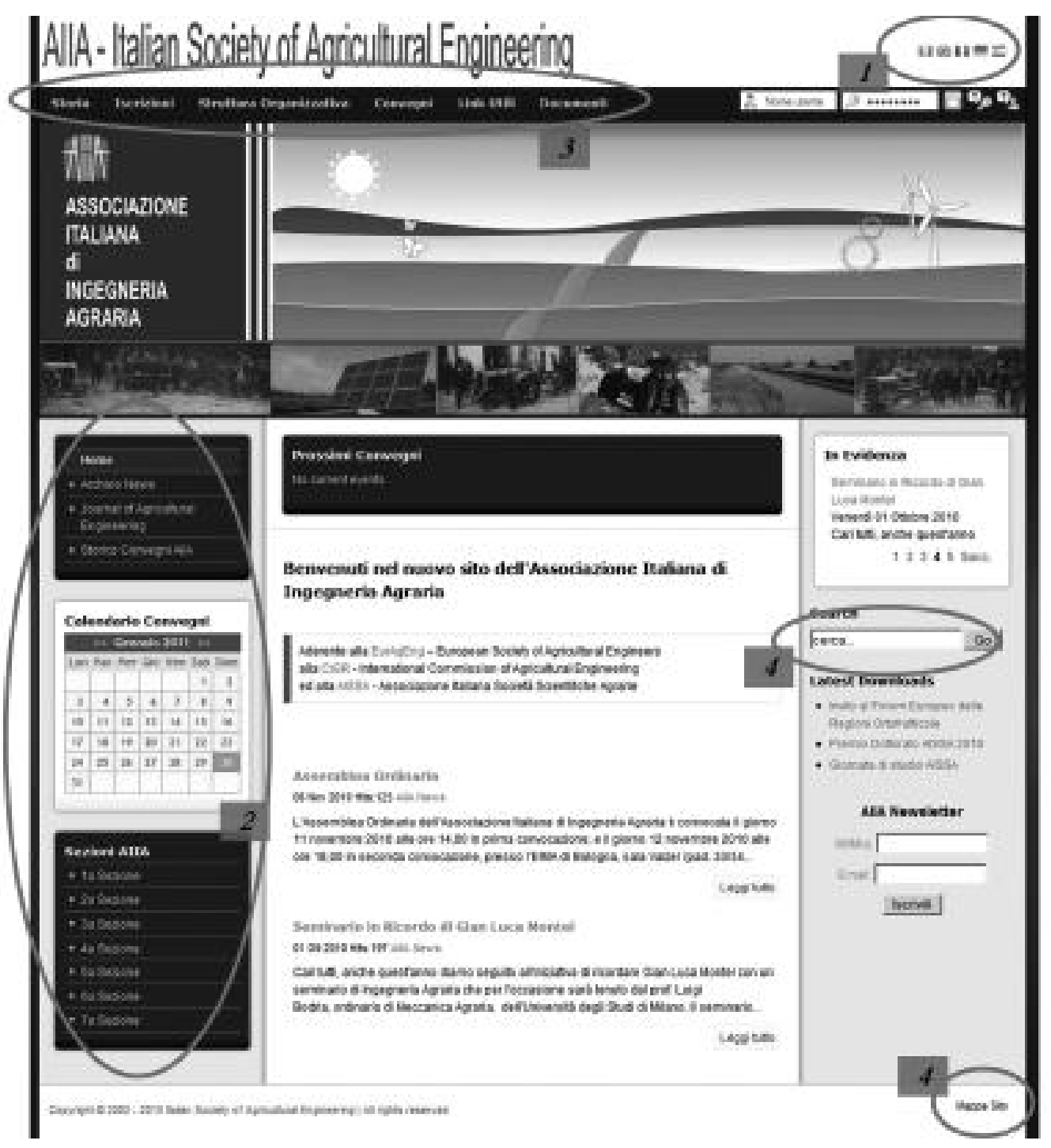

